\title{
Essais
}

Revue interdisciplinaire d'Humanités

\section{Marginália, résister aux limites de l'art dans le Brésil des années 60-70}

\section{Elena Lespes Muñoz}

\section{(2) OpenEdition \\ 1 Journals}

Édition électronique

URL : http://journals.openedition.org/essais/4666

DOI : $10.4000 /$ essais.4666

ISSN : 2276-0970

Éditeur

École doctorale Montaigne Humanités

\section{Édition imprimée}

Date de publication : 15 juillet 2016

Pagination : 142-156

ISBN : 978-2-9544269-8-3

ISSN : 2417-4211

\section{Référence électronique}

Elena Lespes Muñoz, "Marginália, résister aux limites de l'art dans le Brésil des années 60-70 », Essais [En ligne], 9 | 2016, mis en ligne le 23 octobre 2020, consulté le 29 octobre 2020. URL : http:// journals.openedition.org/essais/4666; DOI : https://doi.org/10.4000/essais.4666 


\title{
Marginália, résister aux limites de l'art dans le Brésil des années 1960-1970
}

\author{
Elena Lespes Muñoz
}

"Art vivant, propositionnel, environnemental,
plurisensoriel, conceptuel, artpauvre, artaffluent, rien
de tout cela n'est de l'art. Art vivant, propositionnel,
environnemental, pluri-sensoriel, conceptuel, art
pauvre, art affluent, tout cela est de l'art."

Frederico Morais

Les pratiques artistiques dans le Brésil des années soixante et soixantedix sont marquées par l'expérimentation, celle des formes et des média. Si le questionnement de l'objet d'art ne remonte pas précisément à cette période et lui est antérieur, le premier ready-made de Marcel Duchamp datant de 1913, il n'en constitue pas moins le cœur des réflexions et des expériences menées par les artistes brésiliens d'alors. L'incroyable diversité des formes qui y ont court, et les difficultés d'appréhensions qu'elles posent encore aujourd'hui, témoignent de ce questionnement ontologique de l'art. Sous la forme de documents de performances, de vidéos, de photographies, de poésies visuelles, de notes et de correspondances artistiques, les documents d'art qui nous restent de cette période, posent en effet de nombreuses questions : l'ambiguïté de leur statut, qui repose sur une apparente indifférenciation entre l'œuvre et le document, nous amène à nous interroger sur ce qui échappe à la trace, sur ce qui alors faisait œuvre et qui, aujourd'hui, ne nous parvient que de manière incomplète. L'insistance portée par les artistes brésiliens de cette période sur la reformulation des liens de l'œuvre à son spectateur, dans un rejet évident du regard contemplatif, nous laisse entrevoir l'objet d'une quête, qui n'est pas seulement artistique, mais aussi éthique. 
À cette époque, le Brésil est marqué par un régime dictatorial, celui mis en place lors du coup d'État du 31 mars 1964 par le Maréchal Castelo Branco. Par un discours idéologique fondé sur l'idée d'une "Révolution démocratique " ${ }^{1}$, les militaires se livrent au démantèlement progressif de l'appareil démocratique représentatif et de la classe politique ${ }^{2}$. Aussi, l'analyse de ces pratiques se doitelle d'être contextualisée : sans tomber dans le déterminisme, l'appréhension de ce contexte nous apparaît essentielle pour comprendre la reformulation des liens qu'ont cherché à engager les artistes avec les spectateurs à travers leurs pratiques. La formule de l'artiste Hélio Oiticica "Da Adversidades vivemos" [De l'adversité nous vivons], dans son texte-programme Esquema Geral da Nova Objetividade ${ }^{3}$, figure la prégnance du contexte sur les pratiques artistiques d'alors. Selon la sociologie de l'art développée par Pierre Francastel, les formes esthétiques sont révélatrices d'une expérience sensible du monde en un temps donné sans être pour autant des signes figés et immuables ${ }^{4}$ : le contexte historique constitue ainsi un élément de compréhension de ces formes d'art renouvelées et de leurs mécanismes. Effectivement, l'abandon de la forme traditionnelle de l'œuvre d'art pour ces artistes représente une tentative pour ré-actualiser la relation topique de l'art à la vie. Lautonomie de l'art est délaissée au profit d'une expérience esthétique et sensible où le corps du spectateur se trouve engagé activement. Il ne s'agit pas à la manière de Duchamp de désigner un objet du quotidien pour en faire " une œuvre d'art ", mais plutôt de faire du geste quotidien un geste artistique, une réciprocité qui dans le contexte des années soixante et soixante-dix brésiliennes dépasse la simple dialectique de l'art et de la vie. Le 12 décembre 1968, la journaliste et photographe Marisa Alvarez Lima publie dans la revue O Cruzero, un article intitulé "Marginália - arte e cultura na idade da pedrada ", mentionnant des travaux artistiques liés à la culture marginale 5 . Par extension, «marginália » désigne ici

1 Voir à ce sujet l'article de Maud Chirio, « Le pouvoir en un mot : les militaires brésiliens et la "révolution" du 31 mars 1964 ", Nuevo Mundo Mundos Nuevos [En ligne], colloques, mis en

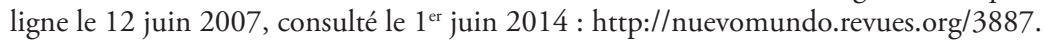

2 Les Actes Institutionnels rythment le durcissement du régime dictatorial : l'AI n 1 (1964) présente les militaires au pouvoir comme l'expression de la souveraineté populaire; l'AI $\mathrm{n}^{\circ} 2$ (1965) impose le bipartisme entre le "parti de la révolution" et le "parti de l'opposition "; l'AI $n^{\circ} 5$ (1968) dissout le Congrès, suspend la Constitution, impose la censure et abroge la plupart des libertés individuelles ; l'AI n ${ }^{\circ} 14$ (1969) rétablit la peine de mort, abolie depuis 1881, etc.

3 Nova Objetividade Brasileira, catalogue de l'exposition, Rio de Janeiro : Musée d'Art Moderne, 1967.

4 "L'œuvre d'art ne propose donc pas à la société des objets figuratifs de ses certitudes antérieures; elle lui offre au contraire des matrices où se révèlent de nouvelles relations, de nouvelles valeurs. ", in Francastel Pierre, La réalité figurative. Éléments structurels de sociologie de l’art, Paris : Éd. Denoël/Gonthier, 1965, p. 19.

5 Parmi ces œuvres, on peut citer Câncer de Glauber Rocha (1968), A margem de Ozualdo de Candeias (1967), O Bandido da Luz Vermelha de Rogério Sganzerla (1968) ; suivront 
cette marge où l'art brésilien, tout en se nourrissant d'une relation créative au quotidien social des grandes villes, a développé des pratiques émancipées des formes traditionnelles de l'art.

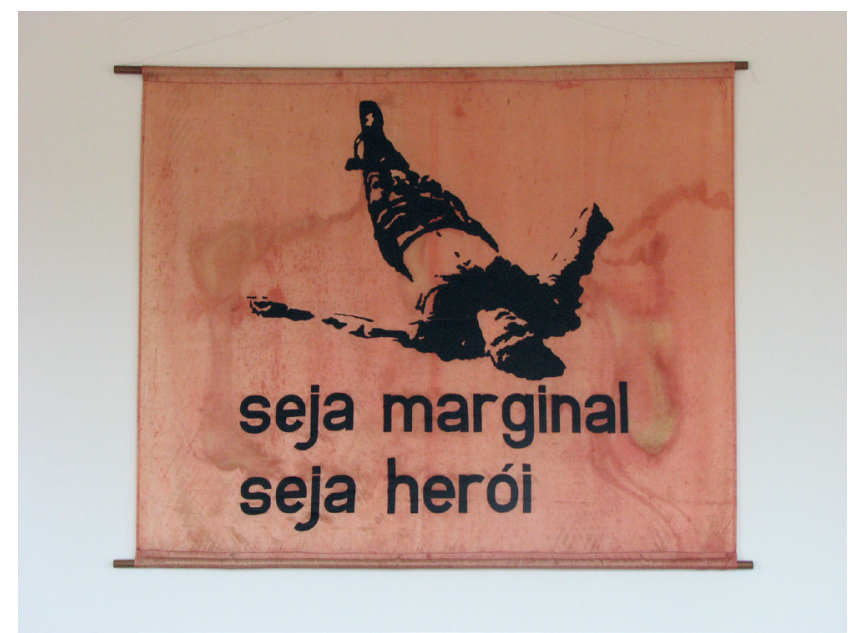

Hélio Oiticica, Seja marginal, Seja Herói, 1968-1996, sérigraphie sur toile, 25/250, $105 \times 90 \mathrm{~cm}$. Courtesy of the artist and Galeria Nara Roesle.

Dans Seja Marginal, Seja Herói, Hélio Oiticica associe ainsi sa condition d'artiste marginal, en marge du marché et des institutions, à celle du célèbre bandit, en marge de la légalité, Cara de Cavalo ${ }^{6}$, assassiné en 1964. Au-delà de son inscription dans une histoire récente du Brésil, la formule de l'artiste fonctionne comme injonction, tout en étant le signe d'un "moment éthique " :

«Souvent, le crime est une sorte de quête désespérée du bonheur. J'ai connu personnellement Cara de Cavalo et je peux dire que c'était mon ami, mais, pour la société, c'était l'ennemi public numéro un (...) Ce qui m’a donné à penser cela a été le contraste entre ce que je connaissais de lui en tant quami, en tant que quelqu'un avec qui je parlais tous les jours comme avec n'importe qui, et l'image crée par la société, ou la façon dont son comportement agissait sur les autres. (...) il est le reflet d'une révolte individuelle contre tous les conditionnements sociaux. " ${ }^{7}$

le livre Urubu-Rei de Gramiro de Mattos (1972) et les textes d'Hélio Oiticica, de Rogério Duarte, de Décio Pignatari, publiés dans des journaux alternatifs tels que Flor do Mal et Presença (Rio de Janeiro, 1971), Verbo Encantado (Bahia, 1974) ou Navilouca (Rio de Janeiro, 1974).

6 Cara de Cavalo (de son vrai nom Manoel Morreira), dont les principales activités étaient le proxénétisme et le jogo do bicho (un type de loterie organisée clandestinement au Brésil) est devenu célèbre suite au meurtre du détective Milton Le Cocq, engagé par un bicheiro pour recouvrer l'argent de paris. Les policiers de Rio de Janeiro créèrent la Scuderie Le Cocq afin de venger la mort de leur compagnon : passant de criminel anonyme à ennemi public $n^{\circ} 1$, Cara de Cavalo sera leur première victime dans leur guerre contre le crime.

7 Oiticica, Hélio, Hélio Oiticica, Exposition Londres : White chapel Gallery, 1969. 
En privilégiant des formes processuelles au profit de la production d'un objet fini, les artistes vont chercher dans le franchissement des limites de ce qui était alors considéré comme de l'art, l'expérimentation d'une liberté alors sanctionnée, cherchant à re-définir les principes d'une conduite active de l'expérience esthétique et éthique.

\section{L'objet d'art délaissé}

Ces pratiques artistiques ont trait au problème de la dématérialisation de l'objet d'art. Par "dématérialisation " de l'objet d'art, il faut comprendre la réduction de sa matérialité à un statut secondaire, voire éphémère et contingent, comme la définit Lucy Lippard dans son texte «La Dématérialisation de l'Art ${ }^{8}$ paru en 1967. Si les pratiques de cette période voient bel et bien l'idée prendre le pas sur l'objet, le terme apparait somme toute réducteur et doit être compris dans un sens métaphorique et non littéral. La matérialité n'est pas absente de ses pratiques, comme en témoignent les traces documentaires laissées par celles-ci. De la même façon, ces expériences résistent à la catégorisation de l'Art Conceptuel, désignant principalement les expérimentations artistiques menées aux États-Unis par Art \& Language, Joseph Kosuth, Sol LeWitt ou encore Lawrence Weiner.

Selon Luis Camnitzer, artiste et critique uruguayen, l'une des caractéristiques principales des artistes d'Amérique du Sud de cette période est d'avoir laissé de côté les questions d'esthétique pour produire des expérimentations qui privilégient la communication et une tentative pour repenser les liens entre l'art et la vie'. Les historiens de l'art latino-américain préferent ainsi au terme d'" Art Conceptuel ", celui de " conceptualismes $»^{10}:$ mis au pluriel, il couvre un champ géographique, esthétique et linguistique plus large. La réduction de l'œuvre à une matérialité "inframince » ne semble en effet pas résulter uniquement d'un processus d'abstraction philosophique, interrogeant le langage et ses structures ${ }^{11}$, mais des conséquences économiques, techniques et politiques engendrées par le

8 Lippard, Lucy et Chandler, John, "The Dematerialization of Art ", écrit en 1967 et publié dans Art international, 12:2, février 1968, p. 31-36. Cité in Six Years: the dematerialization of the art object from 1966 to 1972, Lucy R. Lippard (ed.), Berkeley, Los Angeles, Londres : University of California Press, 1973, p. 43.

9 Camnitzer, Luis, Didactica de la liberación: arte conceptualista latinoamericano, Bogota : Fundación Gilberto Álzate Avedaío, IDARTES, 2012, p. 269.

10 De ce "révisionnisme " critique et historique de l'histoire de l'art, la plateforme de travail d'historiens de l'art latino-américains Red Conceptualismos, fondée en 2007, a fait son sujet, développant ainsi le terme de " conceptualisme». Voir à ce sujet Freire, Cristina ; Longoni, Ana, Conceitualismo do Sul/Sur, São Paulo : Ed. Annablumme, 2009.

11 Je fais ici référence au travail de Joseph Kosuth, ainsi qu’à son texte Art after Philosophy, 1969, in Conceptual art: a critical anthology, Alexander Albero et Blake Stimson (éd.), Cambridge (Mass.), Londres : MIT Press, 1999. 
contexte de la dictature. Le " dépassement du cadre de chevalet $~^{12}$ marque l'exploration de la mise en espace des œuvres et de leurs mises en relation avec le spectateur, ainsi que l'affirmation de la relativité du point de vue et de la visualité. On peut dès lors qualifier de « résistantes » ces productions de l'art brésilien des années soixante et soixante-dix, qui reposent sur une critique sociale, une opposition à des dispositifs de normalisation et une contestation des mécanismes d'encadrements de l'art. Ainsi, pour le poète et critique brésilien Ferreira Gullar, la critique de la circulation en milieu restreint de l'art est nécessaire. Dans Cultura posta emquestão, paru en 1964, celui-ci évoque clairement la nécessité d'un engagement politique de l'art : "si nous rejetons les chemins de l'isolement d'un art puriste ou des fictions fantaisistes, nous devons obligatoirement aborder la question sociale de l'art en termes de pratique politique ", une alternative qui "ne se définit non pas comme une option idéologique, car elle est aussi déterminée par l'évolution historique même du problème artistique dans une société de masse "13. Les artistes ont ainsi cherché à s'émanciper du musée par le déplacement de l'expérience artistique hors de celui-ci ${ }^{14}$. Le refus de l'objet d'art traditionnel répond en un sens à un rejet du système de l'art qui trouve son incarnation dans la fétichisation de l'objet, érigé en bien culturel et symbolique par le musée, et en bien financier par le marché.

Durant cette période, le Grupo REX marque la scène de São Paulo par sa critique du système commercial de l'art et son humour. Formé par les artistes Wesley Duke Lee, Nelson Leirner et Geraldo Barros, le groupe ouvre un espace d'exposition en 1966, la Rex Gallery \& Sons, et crée un périodique, le Rex Time. Le premier numéro du périodique "AVISO: É a guerra » [AVIS : c'est la guerre] est une déclaration de guerre programmatique aux musées, aux galeries, aux critiques et aux biennales, ainsi qu'à l'objet artistique même, réduit selon eux à un bien marchand. Le groupe met fin à ses activités l'année suivante avec Exposição-Não-Exposição [Exposition-non-exposition] : Nelson Leirner annonça dans la presse qu'il offrirait ses œuvres aux premiers arrivants ; l'espace fut vidé en dix minutes ce, malgré les obstacles placés par l'artiste (barres de fer, blocs de ciment, piscine, etc.). On a pu caractériser l'art de cette période, d'art anti-institutionnel malgré l'investissement marqué de certains musées pour présenter ces pratiques contemporaines ${ }^{15}$.

12 Oiticica, Hélio, op. cit., 1967.

13 Gullar, Ferreira, Cultura posta emquestão, Rio de Janeiro : Civilização Brasileira, 1964. Cité dans Amaral, Aracy A., Arte para quê? A preocupação social na arte brasileira 1930-1970, São Paulo : Nobel, 1984, p. 325.

14 Au lendemain de l'année 1968, le milieu de l'art subit de nombreuses répressions, comme en témoigne, au Musée d'Art Moderne de Rio de Janeiro, la fermeture de l'exposition rassemblant les artistes brésiliens qui devaient être présentés à la VI Biennale de Paris. Cette même année, le critique français Pierre Restany initie le boycott de la Biennale de São Paulo en signe d'opposition à la dictature; son appel sera largement suivi.

15 On peut citer le cas du Musée d'Art Contemporain de l'Université de São Paulo, qui sous la 
Dans ce contexte, il est important d'avoir en tête la relation élective entre l'État et la classe privilégiée : au Brésil, cette relation fut traitée par l'historien Réné Armand Dreifuss dans 1964: A conquista do Estado. Ação política, poder e golpe de classe, qui analyse le processus de consolidation des alliances de classe dans les instances du pouvoir : entre la dictature militaire et le capital économique ${ }^{16}$. Le sociologue Marcelo Ridenti parle ainsi de "modernisation conservatrice ${ }^{17}$ : dans sa quête de légitimité, le régime militaire justifia son action autoritaire comme un cadre nécessaire à la reprise économique du pays ; réciproquement, cette légitimité se nourrit du succès d'une modernisation de la société brésilienne engrangé par les mesures économiques et politiques mises en place par le régime avec l'aide d'initiatives privées ${ }^{18}$. Aussi lorsqu'Hélio Oiticica évoque les "nouvelles formules du concept d'anti-art " ${ }^{19}$, il ne s'agit pas tant de rejeter l'art du passé, que de créer de nouvelles conditions pour l'art et l'expérience. Dans une action de rue intitulée Qué é a arte? Para que serve? (1978), l'artiste Paulo Bruscky interroge l'art et sa définition : revêtant les panneaux publicitaires de l'homme-sandwich, il affiche ces questionnements aux passants. Le détournement de la stratégie publicitaire vise à créer un espace spontané de dialogue social par l'usage de la forme interrogative qui invite à délaisser la posture passive du consommateur. L'inquiétude politique transparait au travers de la seconde question, qui pourrait tout aussi bien être "Que peut l'art?".

direction de Walter Zanini (1963-1978), ouvrit le musée à la jeune création contemporaine et à des formes expérimentales alors fort contestées.

16 Dreifuss, René Armand, 1964: A conquista do Estado. Açãopolitica, poder e golpe de classe, Petropolis : Ed. Vozes, 2001.

17 Ridenti, Marcelo, "As oposiçóes à ditadura: resitência e intergração ", p. 7, in $A$ ditadura que mudou Brasil: 50 anos do golpe de 1964, Éd. Motta, Rodrigo Pattosà ; Reis, Daniel Arão ; Ridenti, Marcelo, Rio de Janeiro : Jorge Zahar, 2014. Consulté le 14 octobre 2015 : www.fflch. usp.br/dcp/assets/docs/PDF/Ridenti.pdf.

18 Dans son ouvrage, Ofantasma da revolução brasileira, São Paulo: Unesp, 1993, Marcelo Ridenti souligne que ce désir de modernisation de la société était commun aux opposants de gauche et au régime, ce qui différait étaient les moyens mis en œuvre pour y parvenir.

19 Oiticica, Hélio, op. cit., 1967. 


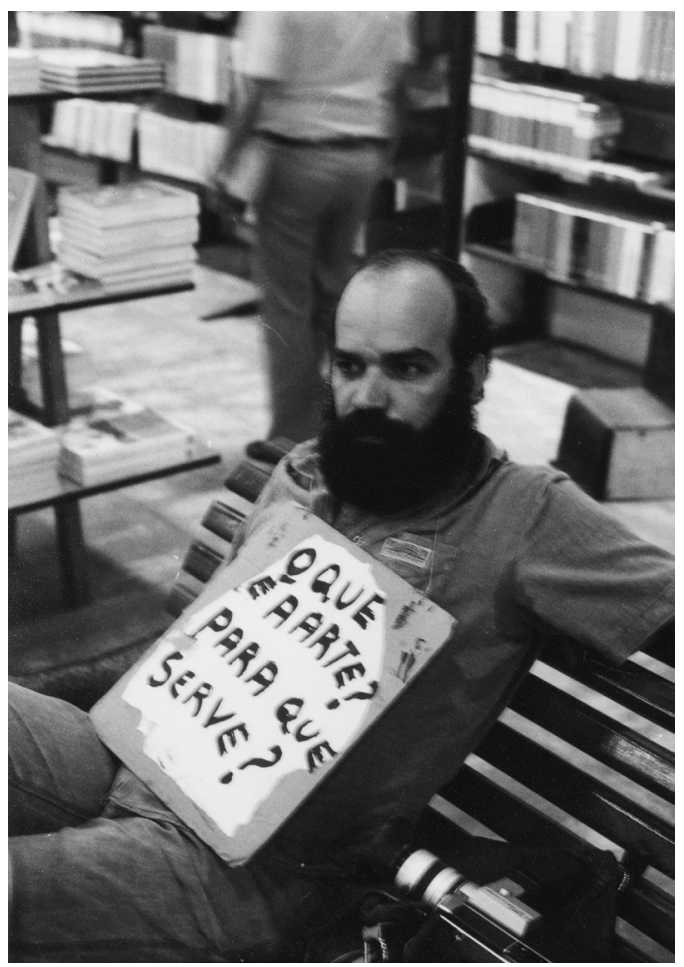

Paulo Bruscky, Qué é a arte? Para que serve?, 1978-2010, photographie Angelo José, ed. 1/3 + 2 PA, 70 x $50 \mathrm{~cm}$, Courtesy of Galeria Nara Roesler.

\section{Occuper le corps}

Sans pour autant porter un message politique explicite, les artistes ont cherché à se compromettre par une expérimentation radicale des langages. Le corps revêt un rôle déterminant dans nombre de ces propositions artistiques. En 1970, l'artiste Antônio Manuel se présente nu devant le jury de la Sélection du Salon d'Art Moderne de Rio de Janeiro. Meu corpo é a obra refuse l'œuvre finie et se présente comme une proposition artistique : l'œuvre est le corps, ou pour aller plus loin, "le corps est le moteur de l'auvre " ${ }^{20}$, il est le vecteur du message, "le corps, et ce qui est en lui, les muscles, le sang, les viscères, les excréments, surtout, l'intelligence $»^{21}$. Le corps de l'artiste se compromet comme œuvre, plus qu'un "morceau d'espace", il devient ce "corps opérant et actuel»"2.

20 Morais, Frederico, "Contra a arte afluente: o corpo é o motor da "obra” ", publié dans la revue Vozes, Rio de Janeiro, janvier/février 1970. Réed. in Arte contemporáneo brasileño: documentos y criticas, Glória Ferreira (coord.), Saint-Jacques de Compostelle : Dardo, 2009, p. 56.

21 Ibid.

22 Merleau-Ponty, Maurice, L'Eil et l'Esprit, Paris : Éditions Gallimard, 1964, p. 16. 
L’artiste Hélio Oiticica développe à partir de 1964, le projet Parangolé2 CEuvre d'anti-art selon les mots de l'artiste ${ }^{24}$, le Parangolé désigne un ensemble de capes, d'étendards, de tentes constitués de matériaux divers et précaires (tissus, plastiques, papier, colle...). Ce projet est né de l'expérience (vivência) de la favela de Mangueira, et de son école de samba, par l'artiste. Pour Hélio Oiticica, la danse permet une désinhibition intellectuelle et la restitution de l'art dans le cadre d'une expérience vivantielle ${ }^{25}$. L'évènement d'art naît de la rencontre du corps du danseur avec la cape et du danseur avec le public ; se développe ainsi un "art environnemental ", éternellement mobile et transformable qui se structure dans l'agir du spectateur, entre port et danse. Les capes et drapeaux ne peuvent donc être exposés comme des œuvres traditionnelles, le parangolé "est plutôt un noyau constructif, laissé ouvert à la participation du spectateur, ce quiest la chose vitale $"{ }^{26}$. Il est ce point de départ dans le sensible, ce point depuis lequel l'expérience et l'ordre ontologique configurent l'œuvre dans l'acte. L'œuvre se déploie dès lors dans le temps, là où la découverte du rythme marque la découverte d'une nouvelle temporalité, celle d'un corps anonyme et collectif à la fois (dans la samba, les gens dansent seuls, mais à l'intérieur d'un groupe et hors de toute chorégraphie préétablie). Le caractère artisanal des capes rend compte de cette puissance de surgissement de l'éphémère : le parangolé reste par définition inachevé et précaire, tout comme les constructions des abris des favelas dont sa structure s'imprègne. Dans cette synthèse de l'abritant et de l'abrité, la corporéité en jeu est celle d'un corps social qui se trouve activé et constitué momentanément dans l'œuvre-évènement.

La reconquête d'un corps social fragmenté et réduit par les violences effectives, mais aussi symboliques, du régime s'opère non seulement dans l'investissement du corps de l'artiste et du spectateur par l'œuvre (et vice-versa), mais aussi dans les stratégies de partages de ces expérimentations artistiques. Le circuit de l'Arte Postal rend compte d'un corps collectif qui se constitue par-delà les frontières physiques de l'espace. De ces échanges d'une grande variété (poésie visuelle, photographies, notes, registres de performances, manifestes, etc.) naît un corps symbolique collectivement constitué, qui offre un régime de visibilité pour des œuvres conçues dans la marginalité. L'Arte Postal repose sur trois critères pleinement réactivés par le médium postal : la communication, l'information et la circulation. Dans ce réseau proprement " rhizomique ", en ce sens qu'il est horizontal, car nourrit non pas d'une base, mais de connexions multiples, la manière même de faire de l'art est bouleversée : interaction, co-création, œuvre

23 À l'origine le mot, issu de l'argot utilisé par les habitants de Rio de Janeiro, désigne des "bavardages ", une situation animée entre différentes personnes.

24 Oiticica, Hélio, Aspiroao Grande Labirinto, Rio de Janeiro : Rocco, 1986, p. 79.

25 J'emprunte ici le terme à Alfonso Caceydo, qui a préféré ce néologisme de l'espagnol vivencia au terme " vécu ", trop passif selon lui.

26 Oiticica Hélio, op. cit., 1969. 
participative, œuvre en processus ou œuvre comme processus, les formes qui y sont pratiquées sont " anti-généalogiques ». La figure de l'artiste est déconstruite au profit d'une figure plurielle et hétérogène. On observe effectivement qu’à travers les expérimentations menées par les artistes de cette période, il ne s'agit pas seulement de "déterritorialiser " l'œuvre et ses espaces d'ostension, en s'émancipant de sa définition traditionnelle et des lieux qui lui étaient dédiés, mais aussi l'unicité de la figure " artiste».

Face à la dictature et à la banalisation de la censure, les artistes sont amenés à questionner l'apparente " vérité » du langage, cet instrument de pouvoir dont ils altèrent les modes et les signes de communication. Ces expérimentations poétiques radicales sont déplacées au sein de nouvelles structures et de formes de communication qui menacent l'entropie du langage : elles se caractérisent par une importante circulation et l'imprévisibilité des échanges. POEMA/PROCESO est un groupe poétique activiste qui revendique alors une radicalisation du langage, critiquant l'expérimentalisme orthodoxe du concrétisme littéraire. Le nom du groupe " PROCESO " souligne une caractéristique fondamentale de leur démarche : aucune production n'est définitive, les lecteurs sont des futurs producteurs. Les artistes s'émancipent de la figure de l'auteur unique. Les membres du groupe, parmi lesquels Wladimir Dias-Pinto, Alvaro de Sá et Neide Dias de Sá, ne cherchent pas seulement à subvertir les canaux traditionnels d'informations, par le biais des revues et du réseau de l'Arte Postal, mais à générer une dynamique de transformation constante pour subvertir la répartition des rôles et affecter l'ordre social. Ils mettent ainsi en place une série d'actions/happenings : le poème spatial $A$ corda (1967), par exemple, consiste en une " corde à linge " tendue sur une place et sur laquelle le participant peut subvertir l'information offerte par la presse papier et élaborer son propre discours à partir d'un ensemble de coupures, de signes verbaux et d'images sélectionnées et suspendues à la corde par le groupe. La corde à linge devient le lieu d'une réécriture où les spectateurs deviennent acteurs en s'appropriant l'ordre narratif de l'actualité et de l'histoire. Le groupe propose une lecture critique et collective dans un espace public sanctionné par la dictature : le titre $A$ corda fait aussi entendre « acorda» qui veut dire « réveille-toi ». 


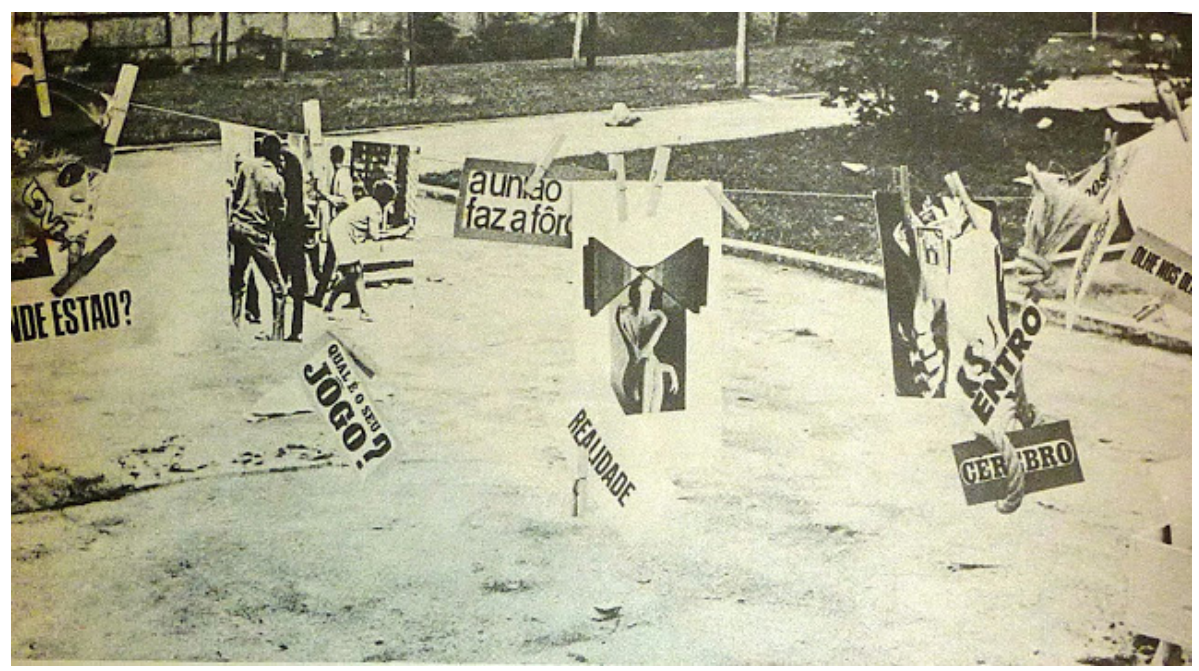

Neide Dias de Sá, $A$ corda, 1967, photographie, 13 x 18 cm. Archive Poema/Processo, Courtesy of the artist.

Cette radicalisation de l'art vers la production collective, que l'on pourrait presque qualifier d'activisme social, provoque une reconfiguration des processus d'émancipation collective qui intègre des stratégies techniques et formelles à la contestation critique.

\section{Radicalité et résistance}

L'expérimentation de formes processuelles par les artistes brésiliens peut dès lors apparaître comme une stratégie ${ }^{27}$ : «Avec l'Acte Institutionnel 5 et la recrudescence de la censure, les artistes furent contraints de trouver des formes d'expression où la référence au social était moins directe. [...] les artistes qui vont rester dans le pays vont chercher dans la marginalité des institutions et l'exacerbation d'une gestualité, une déstabilisation indirecte des valeurs imposées. ${ }^{28}$ Durant cette période, l'art en Amérique du Sud se développe dans une hésitation fondamentale entre le dépassement de l'art (tel qu'il est défini traditionnellement) et une assimilation revendiquée de tout dépassement politique comme étant de l'art. Dans un texte intitulé "Contra a arte afluente: o corpo é o motor da "obra" ", le critique brésilien Frederico Morais pointe les bouleversements qui caractérisent l'art brésilien au début des années soixantedix avec l'avènement de la répression et de la censure politique :

27 Mari Carmen Ramirez parle plus précisément de «stratégies d'anti-discours », in Heterotopias. Medio siglo sin lugar, 1918-1968, Versiones del sur, Madrid : MNCARS, 2000, p. 373.

28 Arantes, Otília, «Depois das vanguardas », in Arte em Revista, São Paulo : Centro de Estudos de Arte Contemporânea, $n^{\circ}$ 7, août 1983, p. 14. 
"L'artiste est aujourd'hui une sorte de guérillero. L'art une forme d'embuscade. Opérant de façon imprévisible, où et quand on l'attend le moins, de manière inusitée [... l'artiste crée un état de tension permanente, une attente constante. Tout peut se transformer en art, même l'évènement quotidien le plus banal. Victime constante de la guérilla artistique le spectateur se voit obligé d'aiguiser et d'activer ses sens [...] et surtout, de prendre des initiatives. $~^{29}$

Ce rapprochement avec la guérilla s'inscrit pleinement dans le contexte de l'époque, où la révolte armée apparut pour beaucoup comme la seule réponse possible aux exactions du régime dictatorial et à laquelle certains artistes prirent part (Sérgio Ferro et Carlos Zíllio ${ }^{30}$ ). À la violence politique exercée par le régime, provoquant une délégitimation, et à laquelle répond la guérilla par une violence d'action effective, nombre d'artistes brésiliens de cette période vont répondre par une violence symbolique. Plaçant leur propre corps au cour de leurs interventions artistiques, franchissant même les limites de leur propre peau, comme Letícia Parente qui cout le mot «Brasil "sur la plante de son pied dans Marca Registrada (1975), certains artistes usent de la violence comme d'une matière artistique propre à fomenter un discours critique efficient. Cette radicalité apparaît comme une stratégie de résistance : la violence performée constituant une forme de théâtralisation de la violence quotidienne, une manière de la mettre à distance tout en l'absorbant.

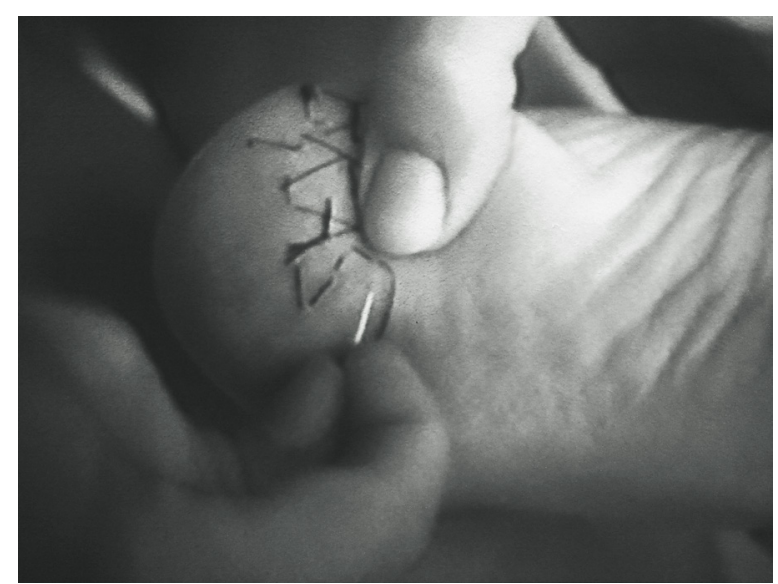

Letícia Parente, Marca Registrada, 1975, 9min. Porta-pack 1/2.

Camera : Jom Tob Azulay. Courtesy of Galeria Jaqueline Martins.

Avec l'exposition "Do corpo a Terra " dans le parc municipal de Belo Horizonte du 17 au 21 avril 1970², le critique Frederico Morais réalise une

29 Morais, Frederico, op. cit., p. 51.

30 Marcelo Ridenti souligne cependant le très faible nombre d'artistes engagés dans la guérilla. Op. cit., p. 73.

31 Participèrent à l'exposition les artistes Artur Barrio, Cildo Meireles, Luiz Alphonsus, Lótus Lobo, Décio Novielo, Dilton Araújo, Eduardo Ângelo, José Ronaldo Lima, Luciano Gusmáo et 
présentation de choc. Malgré le soutien d'Hidrominas (une entreprise de l'État du Mina Gerais), les artistes prirent des risques en expérimentant des formes radicales à la violence et aux messages explicites. En réponse à l'appropriation par le pouvoir en place de la figure historique de Tiradentes ${ }^{32}$, Cildo Meireles présente Tiradentes: totem-monumento ao preso politico. L'artiste amarra dix poules à une planche de bois, les aspergea d'essence et mit soudainement le feu à l'ensemble dans un rituel public d'une rare cruauté. L'acte n'était cependant pas gratuit, en prenant pour matière première de son travail la vie/la mort, l'artiste formulait d'une part la question de la disparition de l'œuvre, mais surtout ne laissait aucun doute quant à son inscription dans un contexte historique extrêmement violent ${ }^{33}$.
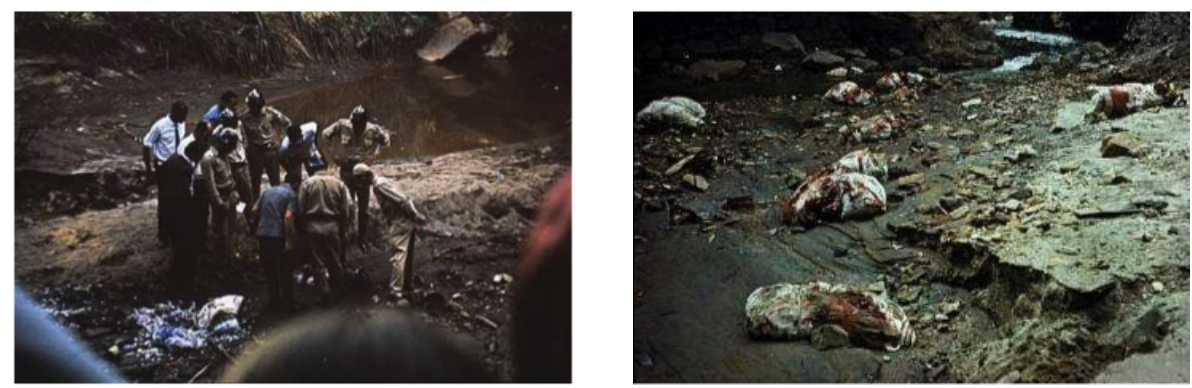

Artur Barrio, Situação T/T, 1 (2 partie), 20 avril 1970, Belo Horizonte, diapositives et photographies PB : César Carneiro, Courtesy of the artist.

Durant cette même exposition, Artur Barrio présente Situação T/T, $1^{34}$. La nuit du 19 avril 1970, l'artiste fabriqua une série de paquets faits de viande en décomposition, d'os et de sang, le tout enroulé dans un tissu et encordé. Le lendemain, il répartissait ces quatorze paquets ensanglantés dans le parc municipal, sur les bords de l'Arrudas. En exhumant symboliquement (mais de manière énigmatique par son geste artistique secret) les cadavres des victimes de la dictature, il s'agissait pour lui de lutter explicitement contre l'indifférence en inquiétant toute perception rassurante, par un véritable défi à l'herméneutique. Véritable disjectamembra où l'artiste fabrique les preuves de l'histoire - tue - de la répression dictatoriale, usant du registre de la métaphore et de l'allégorie.

Lee Jaffe. Selon Antônio Manuel, Hélio Oiticica, Lygia Pape et lui-même furent conviés à participer mais refusèrent.

32 Chef de file de l'« Inconfidence minière " d'Ouro Prêto, accusé de conspiration contre la couronne portugaise et pendu le 21 avril 1792.

33 "Pour rien au monde, je ne répéterai un travail comme Tiradentes (...) mais en 1970, j’ai senti que cela devait être fait. ", Cildo Meireles, dans une interview avec Gerardo Mosqueira, in Herkenhoff, Paulo, Cildo Meireles. São Paulo : Cosac \& Naify, 2000, p. 15.

34 L'œuvre se déroule en trois partie : la fabrication de paquets sanglants, la dispersion de ces paquets sur les rives de l'Arrudas et le déroulage de 60 rouleaux de papier hygiénique à l'endroit où ces paquets furent retirés. 
Tout en étant des "faux ", ces objets mentent moins sur cette histoire qu'ils ne la fabriquent en lui donnant [des] corps. L'artiste crée la trace là où celle-ci est niée, il construit le souvenir. Le trouble public qui s'ensuivit (des rumeurs de crimes commis par les escadrons de la mort circulèrent) fut tel que les sacs furent immédiatement retirés par les forces de l'ordre. En faisant disparaître ces " corps ", les autorités ne faisaient que réitérer un geste de répression politique: sans métaphore aucune, ils faisaient disparaître la source d'un "désordre " ${ }^{35}$. On pressent très bien la force de cette œuvre, malgré le fait qu'elle soit essentiellement contextuelle : ces ballots sont évocateurs dans le contexte brésilien d'alors, quel sens serait le leur une fois déplacé dans le white cube $e^{36}$ ? Si ce n'est devenir la trace d'une trace, capable peut-être d'activer au présent, bien que de façon différente, la charge critique d'un tel évènement. Face à la puissance disruptive de ces expérimentations artistiques, s'offrant au spectateur hors des circuits de l'art et sous des formes "dissensuelles ", la question n'est pas de savoir si ces expérimentations sont (encore) de l'art, mais de mettre en évidence les potentialités critiques et discursives dont elles sont porteuses. Les artistes n'ont pas tant cherché à pousser les spectateurs à l'action qu'à leur permettre de penser autrement, aussi, et comme l'a souligné Jacques Rancière, la frontière entre passivité et activité du spectateur est caduque.

«Les pratiques de l'art ne sont pas des instruments qui fournissent des formes de conscience ou des énergies mobilisatrices au profit d'une politique qui leur serait extérieure. Mais elles ne sortent pas non plus d'elles-mêmes pour devenir des formes d'action politique collective. Elles contribuent à dessiner un paysage nouveau du visible, du dicible et du faisable. Elles forgent contre le consensus d'autres formes de "sens commun", d'un sens commun plus polémique. " ${ }^{37}$

Pour une bonne partie des artistes brésiliens actifs dans les années soixante et soixante-dix, l'art était un espace potentiel de transformation ; peu importe la forme choisie, matérielle ou immatérielle, le lieu où il prenait place, dans la rue ou au musée, l'intérêt portait sur sa capacité à altérer l'état des choses. On ne peut que constater la variété des formes que prirent les expérimentations artistiques dans les années soixante et soixante-dix au Brésil. La dureté du régime, les bouleversements sociaux et la " crise » des institutions artistiques ont participé à l'émergence d'un art confronté aux limites de sa propre définition. En cher-

35 Mesquita, André, Esperarnão é saber: arte entre o silêncio e a evidência, São Paulo : Gráfica Navegar, 2015, p. 59.

36 Le " cube blanc " désigne l'espace de la galerie, et par extension celui du musée. Il est cet espace aux murs blancs fermé qui en un sens garantit l'autonomie de l'art : "Dans ce contexte, un cendrier à pied devient un objet sacré, tout comme un manche d'incendie n'évoque pas tant un manche d'incendie qu'une énigme esthétique ", Brian O'Doherty, "Note sur l'espace de la galerie ", Artforum, 1976. Pour une analyse approfondie, voir Inside the White Cube. The ideology of the Galleryspace, Zurich : JRP Ringier, coll. « Lectures Maison Rouge », 2008.

37 Rancière, Jacques, Le spectateur émancipé, "Les paradoxes de l'art politique ", Paris : La Fabrique, 2008, p. 84 . 
chant à s'émanciper de la censure, ces artistes usent de la transgression esthétique comme détonateur sur le réel, jusqu’à faire de la situation-limite le nouvel espace de l'art. L'expérimentation de "l'art comme action » est ici l'objet d'un questionnement, non seulement épistémologique "Qu'est-ce que l'art ? À quoi sert-il ? ", mais aussi éthique, avec cette question, "Que peut l'art ? ». La dématérialisation, au sens d'action, apparaît alors comme la meilleure économie de cette résistance artistique, ou comme le dit la poète Neide Dias de Sá, du groupe POEMA/PROCESSO : «le signe [c']est l'acte " ${ }^{38}$. Pour résister à " l'esthétisation du politique ", les artistes ont cherché à pratiquer une "esthétique de la disparition ", en rejetant les codes de l'art et l'œuvre d'art elle-même, s'établissant par là tant aux limites de la légalité, que de l'art.

\author{
Elena Lespes Muñoz \\ UMR 8218 Institut Acte \\ Université Paris I \\ GEACC - MAC USP \\ Université de São Paulo, Brésil \\ e.lespes.munoz@gmail.com
}

\begin{abstract}
Résumé
Au Brésil, le coup d'État du 31 mars 1964, mené par le Maréchal Castelo Branco, marque l'avènement de la dictature militaire (1964-1985). Dans le contexte de censure et de répression qui s'en suit, les artistes sont amenés à élaborer des stratégies de contournement, tant dans les pratiques artistiques en elles-mêmes que dans les circuits et les espaces où elles s'expriment. En privilégiant des formes dites "performatives » et en contournant la matérialité de l'art, ces artistes mettent en cause le système de l'art (son objet, comme ses espaces). En étant là où on ne l'attend pas et dans des formes qui lui étaient jusqu'alors étrangères, l'art brésilien de cette période est un art en résistance, car il se joue des limites de sa propre définition et des espaces de libertés restreintes qui lui sont octroyés. Cet article vise à présenter les enjeux de ces déplacements, aussi bien physiques qu'épistémologiques, au travers du travail de certains artistes de cette période (Cildo Meireles, Artur Barrio, Antônio Manuel, etc.)
\end{abstract}

\title{
Mots clés
}

Brésil, art, expérimentation, dictature.

\begin{abstract}
In Brazil, the military coup of March $31^{\text {th }} 1964$, led by Marshal Castelo Branco, marks the begining of the military dictatorship (1964-1985). In this context, artists are compelled to imagine strategies in order to avoid repression and censorship. These strategies affect not only artistic practices themselves, but alsospaces and networks where art used to be presented. Artists discussed the materiality of art and its system by developing "performativ" practices. By beingwhere and when it's not expected, and in unexpected forms, brazilian art of this period
\end{abstract}

38 Neide Dias de Sá, «Uruguay: POESÍA INOBJETAL » (1971), in Padín, Clemente, De la representación a la acción, La Plata, Al Margen, 2010, p. 127. 
in strength: playing with the limits of its own definition and imagining new spaces for liberty. This article will introduce some of the problematics of this period through the works of artists such as Cildo Meireles, Artur Barrio or Antônio Manuel.

\section{Keywords}

Brasil, art, experimentation, dictatorship. 